\title{
Belphégor
}

Littérature populaire et culture médiatique

15-1 | 2017

1936: les Jeux olympiques dans la presse

internationale

\section{Myrtle Cook, une femme à part}

\section{Marilou St-Pierre}

\section{(2) OpenEdition \\ Journals}

\section{Electronic version}

URL: http://journals.openedition.org/belphegor/842

DOI: $10.4000 /$ belphegor.842

ISSN: 1499-7185

Publisher

LPCM

\section{Electronic reference}

Marilou St-Pierre, « Myrtle Cook, une femme à part », Belphégor [Online], 15-1 | 2017, Online since 06 July 2017, connection on 21 April 2019. URL : http://journals.openedition.org/belphegor/842 ; DOI : 10.4000/belphegor.842

This text was automatically generated on 21 April 2019

\section{(c) (i) (3) $\Theta$}

Belphégor est mis à disposition selon les termes de la Licence Creative Commons Attribution - Pas d'Utilisation Commerciale - Pas de Modification 4.0 International. 


\title{
Myrtle Cook, une femme à part
}

\author{
Marilou St-Pierre
}

1 La couverture des Jeux olympiques de Berlin a principalement été une affaire d'hommes. En effet, parmi les journaux dépouillés dans les quatre pays étudiés (France, Belgique, Suisse et Canada), une seule signature de femmes est apparue, celle de Myrtle Cook, chroniqueuse sportive au Montreal Daily Star. Originaire de Toronto, Cook est une ancienne championne olympique qui a remporté l'or au quatre fois 100 mètres lors des Jeux d'Amsterdam en $1928^{1}$. En 1929, elle emménage à Montréal et devient chroniqueuse sportive pour le Montreal Daily Star, où elle continuera à publier sa chronique, In the Women Sportlight, jusqu'en 1969. En plus de son rôle de chroniqueuse, Cook souhaite contribuer à implanter une culture sportive chez les Montréalaises, comme elle le faisait déjà à Toronto. D'ailleurs, elle fera, tout au long de sa carrière dans les médias, une grande place au sport féminin montréalais. Dans ses chroniques, Cook valorise la pratique sportive compétitive chez les femmes, n'y voyant pas une attaque à la féminité, mais simplement la meilleure manière de promouvoir l'activité physique. Ce discours contraste avec celui tenu entre autres par les médecins et les autorités religieuses canadiennes-françaises, qui craignent de voir les femmes se «masculiniser » et voient dans la compétition un reniement de la «nature » féminine, fragile et peu compétitive. Pour être acceptée, la pratique sportive féminine devrait avoir pour finalité la préparation des femmes à leur future vie de mères et d'épouses. De son côté, Cook invite plutôt les femmes à transgresser «le modèle traditionnel de la féminité ${ }^{~}$ et réfute les arguments biologiques à l'égard de la fragilité naturelle des femmes. À travers ses écrits, elle propose une conception du sport qui agit comme un vecteur d'égalité entre les hommes et les femmes.

2 Ce discours engagé de Cook et l'importance qu'elle accorde à la compétition chez les femmes sont saillants dans sa couverture des Jeux olympiques de Berlin. En fait, Cook apparait souvent comme une voix discordante parmi les discours véhiculés par les journaux montréalais. Cette discordance s'exprime dans un premier temps par le sujet même de sa chronique, soit les athlètes olympiques féminines. En effet, dans la couverture générale des JO, les sports féminins pratiqués sont marginalisés ${ }^{3}$. Ils ne font pas l'objet de grands titres, se retrouvent le plus souvent à la fin des articles principaux et 
les résultats masculins sont systématiquement présentés avant les résultats féminins. Quant aux chroniques portant sur les JO, à l'exception de quelques lignes qui soulignent la beauté des Canadiennes ou la sévérité d'Avery Brundage envers Eleanor Holm Jarrett, les enjeux du sport féminin y sont ignorés. Or, dans les chroniques de Myrtle Cook, le sport féminin prend toute la place. En fait, Cook y livre souvent des résumés très détaillés des compétitions, à tel point que par moment, le lecteur peut croire qu'elle est elle-même à Berlin. Ces résumés des épreuves mettent l'accent sur les réalisations et les déceptions des athlètes, ainsi que sur les prouesses physiques qu'elles déploient. Nous ne trouvons rien de comparable dans les pages des journaux montréalais ${ }^{4}$, ni sous la plume d'un homme ni sous celle d'un auteur anonyme.

3 L'ancienne médaillée olympique utilise également l'espace qui lui est alloué dans le Star pour faire valoir sans équivoque que les femmes ont tout autant le droit que les hommes de se trouver sur une piste de course ou dans une piscine olympique et que la place des femmes n'est pas qu'à la cuisine. Ainsi, dans une chronique datée du 7 août 1936, Cook va de cette charge contre Mussolini et une certaine frange de la société italienne :

Now that one of his fair subjects has captured an Olympic title, Mussolini may not be as set against women in competition - we gathered from numerous speeches that he preferred them in the home tending to their knitting and keeping the kitchen spick and span. Now that Miss Valla has proven to the world that the Italian girls can compete with the best of them, the attitude that is general towards competitive sport for women in her country may well change - her triumph will focus deep interest on their women athletes at any rate 5 .

Dans cette citation, nous voyons Cook opposer une image traditionnelle de la féminité, celle de la femme au foyer, s'occupant de garder la maison propre et en ordre, à un autre modèle, celui de l'athlète compétitive, capable de briller par ses performances et de triompher pour son pays. Un modèle qui ne s'applique par seulement aux Canadiennes, mais également aux femmes d'autres pays, tel l'Italie.

Dans une autre chronique, Cook vilipende ouvertement une ancienne athlète américaine, Olive Hatch

who believes that you do not get value received from amateur athletics once you reach the top bracket! Chatting with a correspondent at Hollywood yesterday, she stated she was glad she was out of it! [...]. She is in the movies now and through forever with competitive athletics - claims she has a real career - thinks women athletes cannot come back - hard enough for a man, but virtually impossible for a woman, she thinks. It has been done Olive! What better example can we cite than Mrs Helen Wills Moody ${ }^{6}$.

Elle s'attaque encore une fois à une image stéréotypée des femmes, qui seraient incapables de reprendre la compétition après une pause dans leur carrière. Le fait qu'Hatch soit elle-même une femme ne semble pas infléchir le point de vue de Cook : les femmes peuvent autant que les hommes revenir à la compétition et atteindre de nouveau les sommets.

7 Les paroles de Hatch mettent tout de même en relief un fait qui n'est pas complètement contredit par Cook, soit le peu de valeur accordée aux athlètes féminines qui atteignent les sommets de leur sport. On devine, vu la ponctuation utilisée par Cook, qu'elle n'est pas complètement en accord avec cette affirmation, mais ce désaccord n'est pas exprimé explicitement. La couverture des JO par les journaux laisse croire qu'en effet, les femmes, même lorsqu'elles atteignent les sommets, ne reçoivent pas une grande attention. En fait, ce sont les scandales qu'elles soulèvent (féminité contestée d'Helen Stephens, expulsion 
de Holm Jarrett) qui attirent le plus l'attention, et non leurs performances, sauf bien entendu de la part de Cook elle-même.

\section{L'impact d'une chroniqueuse sur la couverture des Jeux}

Que change la présence d'une femme dans la couverture des JO de Berlin? Bien entendu, nous ne pouvons simplement affirmer, sous peine de sombrer dans une forme d'essentialisme primaire, que la seule présence d'une femme dans une rédaction sportive change complètement la donne. Mais on peut affirmer que la présence de Myrtle Cook, une femme engagée qui ne craint pas les sujets polémiques, suscite des débats et des angles de couverture qui, sinon, seraient restés lettre morte. Au delà de son traitement journalistique du sport féminin - et même si cette pratique est, en soi, loin d'être commune - elle questionne de front certains discours dominants, comme en font foi les exemples précédents, ainsi que sa défense d'Helen Stephens et de la Fédération sportive féminine internationale ${ }^{7}$. Elle critique également des discours diffusés par son propre journal, le Star, ou par des journalistes qui apparaissent parfois dans ses pages. Ainsi, le 5 août, Cook réagit à l'affirmation selon laquelle "women discuss throwers resemble nothing so much as minsky burlesque girls overcome by age and fat ${ }^{8} »$. Ces mots,

that bounced off Henry McLemore ${ }^{9}$ typewritter and to our way of thinking are harsh and unjust. We have in our many travels seen and watched in action some very fine and attractive woman discus throwers- Jadwiga Waja of Poland is not hard to look at - in repose Gisela Mauermayer who won the Olympic title yesterday with a too of 156 feet $33 / 8$ inches to shatter the Olympic record is a stately attractive girl - Lillian Copeland of U.S.A who formerly held the Olympic record, and who is now a practicing lawyer, is also a lovely gir ${ }^{10}$.

9 Si la chroniqueuse anglophone défend la féminité des athlètes, que ce soit en soulignant leur beauté ou leurs qualités "féminines", et semble se rallier ici à une vision plutôt stéréotypée des rôles sexués, elle demeure une voix discordante qui adopte un point de vue militant unique dans la couverture des Jo de la presse montréalaise. Myrtle Cook est sans contredit à l'origine d'un discours féministe sur le sport, à l'intérieur de pages sportives qui sont loin de pouvoir porter ce titre.

\section{NOTES}

1. Les informations biographiques sur Cook sont issues de Detellier, Élise, Mises au jeu : Les sports féminins à Montréal, 1919-1961, Montréal, Les Éditions du Remue-ménage, 2015, 300 p.

2. Detellier, $2015:$ p. 82.

3. Voir dans ce numéro, « Genre, Race et Nation ».

4. Notons toutefois que Myrtle Cook n'est pas la seule femme à bénéficier d'une tribune dans les pages sportives au Canada. En effet, au Canada anglais, plusieurs journaux comptent une chroniqueuse sportive dans leur rang, dont le Toronto Star, le Globe and Mail, le Winnipeg Free Press, etc. (Detellier, $2015: 69$ ). Ce n'est toutefois pas le cas pour les journaux montréalais. 
5. Cook, Myrtle, «In the Women Sportlight ", The Montreal Daily Star, 7 août 1936, p. 18. (« Maintenant que l'une de ses sujets a remporté un titre olympique, Mussolini ne peut pas être opposé aux compétitions féminines - nous avons retracé plusieurs discours dans lesquels il dit préférer les femmes qui restent à la maison, à tricoter et à garder la cuisine propre. Maintenant que Mlle Valla a prouvé au monde que les filles italiennes peuvent compétitionner avec les meilleures, l'attitude générale du pays par rapport au sport féminin peut changer - son triomphe attirera l'attention sur les athlètes féminines » (notre traduction)

6. Cook, Myrtle, «In the Women Sportlight ", The Montreal Daily Star, 6 août 1936, p. 25 ; " qui croit que vous ne recevez pas de signe d'estime de la part du sport amateur une fois que vous avez atteint le sommet! S'adressant à un correspondant hier à Hollywood, elle a dit être heureuse de ne plus être dans ce champ d'activité. [...] Elle est maintenant dans l'industrie du film et en a terminé avec la compétition sportive - elle a maintenant une vraie carrière - et elle pense que les femmes athlètes ne peuvent pas faire de retour à la compétition - difficile pour un homme, mais virtuellement impossible pour une femme. Mais cela a été fait olive! Quelle meilleure exemple peut-on citer que celle de madame Helen Wills Moody " (notre traduction).

7. Voir dans ce numéro l'article « Race, genre et nation ».

8. Soit : « les femmes qui compétitionnent au lancer du poids ne ressemblent à rien d'autres qu'à des filles burlesques accablées par l'âge et la graisse » (notre traduction)

9. Henry McLemore, correspondanr de la United Press, couvre les Jeux à partir de Berlin. Le Star a publié certains de ses textes durant les deux semaines de compétition.

10. Cook, Myrtle, «In the Women Sportlight », The Montreal Daily Star, 5 août 1936, p. 20 ; ces mots « qui ont bondi de la machine à écrire d'Henry McLemore sont selon nous durs et injustes. Lors de nos nombreux voyages, nous avons vu plusieurs femmes jolies et attirantes pratiquer le lancer du poids - Jadwinga Wafa de Pologne, n'est pas désagréable à regarder - au repos, Gisela Mauermayer, qui a remporté le titre olympique hier avec un lancée de 156 pied et $33 / 8$ pouces, brisant le record olympique, est une fille majestueuse - Lillian Copeland des États-Unis, qui possédait le précédent record olympique et qui pratique maintenant le droit, est également une fille ravissante » (notre traduction)

\section{ABSTRACTS}

La couverture des Jeux olympiques de Berlin n'a pas fait exception à la règle en matière d'actualité sportive, alors qu'on ne dénombre qu'une seule femme attitrée au suivi des JO. En effet, Myrtle Cook, chroniqueuse sportive au Montreal Daily Star, signe tout au long des deux semaines de compétition une chronique quotidienne où elle relate en détail les hauts faits d'armes des athlètes féminines, se démarquant par le fait même, de l'ensemble des journaux, où la présence des sportives est généralement marginalisée. Cook, ancienne championne olympique et fière défenseure de la pratique sportive chez les femmes, ne s'arrête pas seulement au résumé des compétitions. Par des prises de position fortes, la chroniqueuse anglophone conteste le discours dominant, osant remettre en cause le discours biologique sur la faible "nature » des femmes et les stéréotypes dont les athlètes féminines sont affublées. Si la présence d'une femme ne modifie pas dramatiquement la couverture des Jo de Berlin, elle contribue toutefois à faire émerger un contre-discours et des enjeux qui autrement, seraient passés sous silence. 


\section{INDEX}

Mots-clés: 1936, presse, Myrtle Cook, chronique, athlètes féminines, jeux olympiques, sport, médias, culture médiatique, Montreal Daily Star, stéréotypes féminins

\section{AUTHOR}

\section{MARILOU ST-PIERRE}

Marilou St-Pierre est candidate au doctorat en communication à l'Université Concordia.

Publications : Charron Jean, Marilou St-Pierre et Geneviève Drolet. 2015. « La parole des femmes dans les journaux télévisés au Québec, 1961-2010 », Communication [En ligne], vol. 33/1 | 2015, mis en ligne le 16 février 2015. URL : http://communication.revues.org/5127 ; DOI : 10.4000/ communication.5127. « Women Sports Reporters : Feminity in a traditional male field ». Dans II International Conference Gender and Communication, Conference proceeding, 2014, p. 50-63. Brin, Colette et Marilou St-Pierre. «Crise des médias et effectifs rédactionnels au Québec », Centre d'études sur les médias, 2013, 60 p. 